\title{
Age Effects on Cortical Thickness in Cognitively Normal Elderly Individuals
}

\author{
Sona Hurtz ${ }^{a}$ Ellen Woo ${ }^{a}$ Valeria Kebets ${ }^{d}$ Amity E. Green ${ }^{c}$ \\ Charleen Zoumalan $^{a}$ Benjamin Wang ${ }^{a} \quad J o h n$ M. Ringman ${ }^{a}$ \\ Paul M. Thompsona, biana G. Apostolova ${ }^{a}$ \\ a Department of Neurology, University of California Los Angeles, and b ${ }^{b}$ Laboratory of \\ Neuro Imaging, University of Southern California, Los Angeles, Calif., USA; ' Department of \\ Psychology and Psychiatry, Monash University, Melbourne, Vic., Australia; ${ }^{d}$ University of \\ Geneva, Geneva, Switzerland
}

\section{Key Words}

Normal aging · Imaging · Atrophy · Cognition · Magnetic resonance imaging

\section{Abstract}

Background/Aims: Atrophy in both grey and white matter is found in normal aging. The prefrontal cortex and the frontal lobe white matter are thought to be the most affected regions. Our aim was to examine the effects of normal aging on cortical grey matter using a 3D quantitative cortical mapping method. Methods: We analyzed 1.5-tesla brain magnetic resonance imaging data from 44 cognitively normal elderly subjects using cortical pattern matching and cortical thickness analyses. Linear regression analysis was used to study the effect of age on cortical thickness. 3D map-wide correction for multiple comparisons was conducted with permutation analyses using a threshold of $p<0.01$. Results: We found a significant negative association between age and cortical thickness in the right hemisphere $\left(p_{\text {corrected }}=0.009\right)$ and a trend level association in the left hemisphere $\left(p_{\text {corrected }}=0.081\right)$. Age-related changes were greatest in the sensorimotor, bilateral dorsal anterior cingulate and supplementary motor cortices, and the right posterior middle and inferior frontal gyri. Age effects greater in the medial than lateral visual association cortices were also seen bilaterally. Conclusion: Our novel method further validates that normal aging results in diffuse cortical thinning that is most pronounced in the frontal and visual association cortices.

(C) 2014 S. Karger AG, Basel 


\section{Introduction}

According to the Department of Health and Human Services, the aging population in the United States will grow to an estimated 72.1 million by 2030, more than twice the number of older adults in 2000 [1]. Therefore, it is imperative to prepare to foster the wellbeing of older adults. Currently, many studies investigating cognitive and functional changes in normal aging are being conducted. Researchers agree that the degree with which cognition is affected varies from one individual to another [2], and this variability may in part reflect incipient disease processes in a subset of individuals. Aging is commonly associated with loss of structural integrity of the cortical grey matter and expansion of the ventricles due to loss of tissue. The most commonly reported cortical region that is affected in aging is the prefrontal cortex, an area responsible for attention and executive functions [3-7].

A number of studies have investigated the effects of aging on cerebral grey matter using advanced neuroimaging techniques. By far the most widely replicated and arguably most susceptible cortical region seems to be the frontal cortex [5, 6, 8-11], although associations with other brain regions have been reported including the parietal [5-9] and temporal [6, 8, 9] cortices, the primary motor and sensory cortices $[9,11]$, the secondary visual cortex $[6,8]$, and the anterior cingulate [9].

A better understanding of these age-associated changes of the human cortex will advance our knowledge of the normal aging process and help to distinguish it from the neurodegenerative effects of many neurological disorders of the elderly, such as Alzheimer's disease (AD). The aim of our study was to assess the effects of age on cortical thickness using another well-established quantitative cortical mapping method in a well-defined cognitively normal elderly cohort.

\section{Materials and Methods}

\section{Subjects}

We analyzed SPGR (spoiled gradient echo) T1-weighted magnetic resonance imaging (MRI) data for 44 cognitively normal elderly controls from the University of California, Los Angeles (UCLA) Alzheimer's Disease Research Center database, an ongoing longitudinal study of $\mathrm{AD}$, other dementias, and normal cognitive aging. All participating subjects provided informed consent following the Declaration of Helsinki and the restrictions and policies of the UCLA Institutional Review Board.

Demographic and cognitive data were collected through detailed neurological examinations, interviews as well as a comprehensive neuropsychological evaluation including the following tests: Mini-Mental State Examination (MMSE) [12], the Wechsler Adult Intelligence Scale - 3rd edition (WAIS-III: 8 subtests) [13], the Boston Naming Test [14], the Controlled Oral Word Association Test (FAS and Animals) [15], the Wechsler Memory Scale - 3rd edition (WMS-III: Logical Memory I and II and Visual Reproduction I and II) [16], California Verbal Learning Test - 1st or 2nd edition (CVLT-I or CVLT-II) [17, 18], the Rey-Osterrieth Complex Figure test - copy and 3-min recall (ROCFT) [19], the Stroop Interference Test [20], Trailmaking A and B [21], and the Wisconsin Card Sorting Test-64 (WCST-64) [22]. Diagnosis was reached by consensus among neurologists, psychiatrists, and neuropsychologists. Normal aging was defined as scoring within normal limits based on age- and education-adjusted norms on a predefined subset of the tests from the neuropsychological battery. Exclusion criteria included age $<50$, history of significant drug or alcohol abuse, and concurrent psychiatric or neurological illness. 


\section{Image Acquisition and Analysis}

Structural SPGR T1-weighted images were acquired using a 1.5-tesla Signa scanner (GE Medical Systems, Milwaukee, Wisc., USA) with the following protocol: gapless coronal acquisition with $28 \mathrm{~ms}$ repetition time, $6 \mathrm{~ms}$ echo time, $220 \mathrm{~mm}$ field of view, $256 \times 192$ voxels in acquisition matrix, and $1.5 \mathrm{~mm}$ slice thickness. The MRI scans were intensity normalized using a regularized tricubic B-spline approach and spatially scaled using the Minctracc algorithm and a 9-parameter linear transformation ( 3 translations, 3 rotations, and 3 scales) to fit the International Consortium for Brain Mapping 53 (ICBM53) average brain imaging template [23, 24].

An intracranial mask of the brain was generated using an automated brain surface extraction algorithm tool in BrainSuite [25]. The outputs were visually inspected and all segmentation errors (i.e., mislabeled brain or nonbrain tissue) were manually corrected. Hemispheric masks were produced. Bias field correction was applied to correct intensity errors due to magnetic field inhomogeneities. Each voxel was classified as cerebrospinal fluid, grey matter, and white matter based on signal intensity values [25]. The grey matter data were interpolated in $0.33 \mathrm{~mm}$ isotropic 'subvoxel' resolution. Resampling of the maps to subvoxel spatial resolution reduces subsequent inaccuracies in computing thickness fields by calculating distances to sets of voxels. After automated 3D hemispheric reconstruction, 39 sulci per hemisphere were traced following a well-established protocol with high interrater reliability [26]. All individual sulcal maps were averaged to create a representative studyspecific sulcal map. Next, cortical surfaces were parameterized, flattened, and warped to align all individual sulci with the average sulcal models assuring that all analogous gyri were as explicitly matched as possible [27] (fig. 1). Cortical grey matter thickness (the distance in millimeters between the grey/white and the grey/cerebrospinal fluid boundaries) was computed at each surface point and smoothed using a surface-based kernel with $10 \mathrm{~mm}$ radius [28].

Linear regression analysis was conducted with age as the predictor variable and cortical thickness as the outcome variable. Both statistical significance and correlation maps were created to calculate the effect of age on cortical thickness at every surface point. We investigated this association by correcting for multiple comparisons using permutation testing with a threshold of $\mathrm{p}<0.01$. In our permutation analyses, we created 100,000 random simulations in which we computed the fraction of experiments that would be observed by chance. These methods are standardized and have been described in greater detail elsewhere [29].

\section{Results}

Our sample consisted of 26 males and 18 females with a mean age of $66.09 \pm 7.23$ years (range: $51-79$ ), a mean education of $17.25 \pm 2.40$ years (range: $13-24$ ) and an average MMSE score of $29.34 \pm 0.78$ (range: $27-30$ ). Follow-up data were available for 37 subjects (mean duration of follow-up $6 \pm 4$ years, range: 1-11). Of these 37, 2 subjects had converted to dementia in years 2 and 10 of follow-up. Our findings were reported for all subjects and also after excluding the 2 subjects who later converted to dementia. The mean cortical thickness for the final cohort was $2.5 \mathrm{~mm}$ for the right hemisphere and $2.6 \mathrm{~mm}$ for the left hemisphere, which corresponds well with the previously reported range of 1.5-3.4 $\mathrm{mm}$ [30].

In figure 2, the statistical and correlation maps show the relationship between age and cortical thickness in our elderly adult cohort. The global permutation-corrected significance of the association between age and cortical thinning was $\mathrm{p}_{\text {corrected }}=0.009$ for the right and $\mathrm{p}_{\text {corrected }}=0.081$ for the left hemisphere. The cortical areas showing significant associations included the sensorimotor [Brodmann area (BA) 1-4], supplementary motor cortices (medial 


\section{Registration to ICBM53 space}

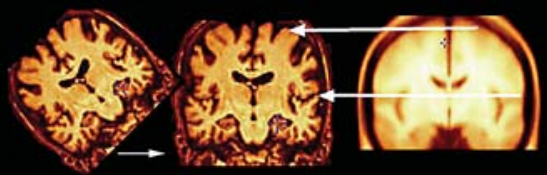

\section{Skullstripping, 3D hemispheric} reconstruction, and sulcal line tracing
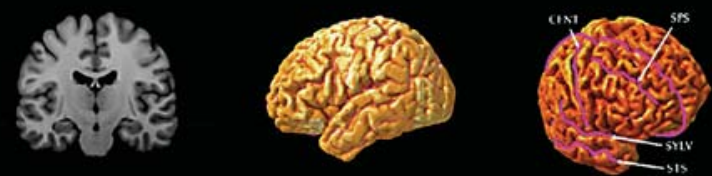

Cortical surface flattening and warping

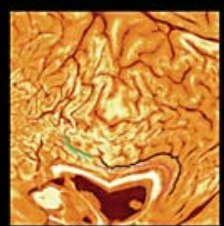

\section{Construction of parametric meshes}

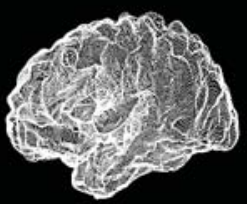

Grey matter mapping

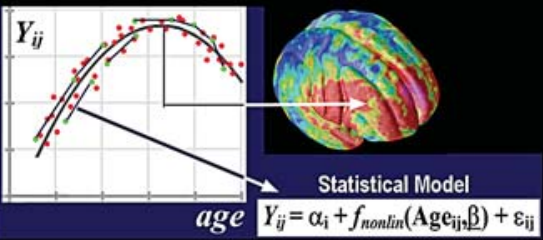

Fig. 1. Schema of cortical pattern matching method.

BA 4 and 6), dorsal anterior cingulate (BA 24), posterior inferior temporal (BA 37) and visual association cortices (BA 18-19) bilaterally, as well as the premotor (lateral BA 4 and 6) and the posterior parts of the prefrontal cortex (BA 8, 9 and 44) on the right, and the precuneus/ posterior cingulate (BA 23 and 31) and the primary visual cortex (BA 17) on the left. Of note, the entorhinal/parahippocampal area - the cortical region affected earliest by Alzheimer's pathology - did not show significant age-associated atrophy. This area showed a nonsignificant effect in the opposite direction. After excluding the two normal control subjects who later converted to dementia, our maps remained largely unchanged; therefore, only the maps 
Fig. 2. Statistical (left) and correlation maps (right) demonstrating the association between age and cortical grey matter thickness in cognitively normal subjects.

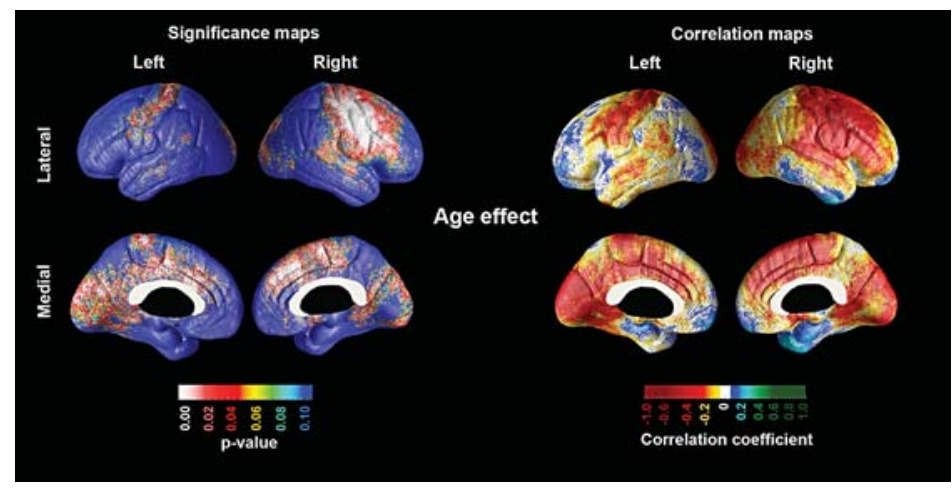

from the entire cohort are shown. The map-wise permutation-corrected significance of the maps after excluding the 2 subjects who progressed to dementia during the follow-up period were $\mathrm{p}_{\text {corrected }}=0.013$ for the right and $\mathrm{p}_{\text {corrected }}=0.089$ for the left hemispheres.

\section{Discussion}

Our findings suggest that the different divisions of the cortex have differential susceptibility to aging, both anatomically and phylogenetically. In general, we found that the neocortical areas were profoundly affected, while the allocortical areas were largely preserved. This pattern is distinct from the one observed in AD in two very important ways. Our work and the work of others clearly show that aging has a significant impact on the primary sensory, motor, and visual cortices, which are relatively spared in $\mathrm{AD}$, while at the same time normal aging spares the entorhinal/parahippocampal regions, which are affected earliest in AD. Our findings extend the findings of other research studies $[2-9,11]$ by capturing a distinct pattern of cortical thinning with advancing age using a novel cortical mapping method. By doing so, our study improves our understanding of the structural effects of aging on the brain. Such work is important, as it will allow us to more insightfully interpret future imaging studies, especially when considering cognitively normal subjects with positive amyloid positron emission scans. This is an important problem given that approximately one third of all cognitively normal elderly have positive amyloid scans [31], and it remains unclear who of these subjects will progress to dementia and who will not. In these cases, determining whether these subjects have atrophy in the primary sensory, motor, and visual cortices versus the entorhinal/parahippocampal regions might provide an important disease biosignature to determine a better prognosis.

Several strengths and limitations of our study should be recognized. Although the majority of subjects had longitudinal clinical data and therefore were not at imminent risk for clinical dementia, the imaging analyses were cross-sectional. A longitudinal approach would be even more informative with potential to show the trajectory of gradual age-associated structural changes in the brains of elderly individuals over time. The relatively small sample size and narrow age variability in our cohort are additional limitations of our study. However, the narrow age span and relatively younger mean age of this cohort allowed for the examination of earlier aging effects that are quite distinct from what is commonly seen in $\mathrm{AD}-\mathrm{a}$ disease with exponential increase in prevalence with age. Despite these limitations, this study was strengthened by the use of explicit cortical morphology mapping and advanced imaging software allowing for the detection of subtle structural effects associated with normal aging. 


\section{Acknowledgements}

This study was generously supported by NIA K23 AG026803 (jointly sponsored by NIA, AFAR, The John A. Hartford Foundation, the Atlantic Philanthropies, the Starr Foundation and an anonymous donor), NIA P50 AG16570, and the Easton Consortium for Alzheimer's Disease Drug Discovery and Biomarker Development as well as R01 EB008281 (No. 14).

\section{Disclosure Statement}

The authors have no conflicts of interest to disclose.

\section{References}

1 Aging Statistics. Administration on Aging - Department of Health and Human Services, 2011. http://www.aoa. gov/aoaroot/aging_statistics/index.aspx.

-2 Hillman CH, Erickson KI, Kramer AF: Be smart, exercise your heart: exercise effects on brain and cognition. Nat Rev Neurosci 2008;9:58-65.

-3 Gunning-Dixon FM, Raz N: Neuroanatomical correlates of selected executive functions in middle-aged and older adults: a prospective MRI study. Neuropsychologia 2003;41:1929-1941.

-4 Head D, Rodrigue KM, Kennedy KM, Raz N: Neuroanatomical and cognitive mediators of age-related differences in episodic memory. Neuropsychology 2008;22:491-507.

5 McGinnis SM, Brickhouse M, Pascual B, Dickerson BC: Age-related changes in the thickness of cortical zones in humans. Brain Topogr 2011;24:279-291.

-6 Raz N, Gunning FM, Head D, Dupuis JH, McQuain J, Briggs SD, Loken WJ, Thornton AE, Acker JD: Selective aging of the human cerebral cortex observed in vivo: differential vulnerability of the prefrontal gray matter. Cereb Cortex 1997; 7:268-282.

7 Salat DH, Kaye JA, Janowsky JS: Greater orbital prefrontal volume selectively predicts worse working memory performance in older adults. Cereb Cortex 2002;12:494-505.

-8 Fjell AM, Westlye LT, Amlien I, Espeseth T, Reinvang I, Raz N, Agartz I, Salat DH, Greve DN, Fischl B, Dale AM, Walhovd KB: High consistency of regional cortical thinning in aging across multiple samples. Cereb Cortex 2009;19:2001-2012.

-9 Good CD, Johnsrude IS, Ashburner J, Henson RN, Friston KJ, Frackowiak RS: A voxel-based morphometric study of ageing in 465 normal adult human brains. Neuroimage 2001;14:21-36.

10 Jernigan TL, Archibald SL, Fennema-Notestine C, Gamst AC, Stout JC, Bonner J, Hesselink JR: Effects of age on tissues and regions of the cerebrum and cerebellum. Neurobiol Aging 2001;22:581-594.

11 Salat DH, Buckner RL, Snyder AZ, Greve DN, Desikan RSR, Busa E, Morris JC, Dale AM, Fischl B: Thinning of the cerebral cortex in aging. Cereb Cortex 2004;14:721-730.

12 Folstein MF, Folstein SE, McHugh PR: 'Mini-mental state'. A practical method for grading the cognitive state of patients for the clinician. J Psychiatr Res 1975;12:189-198.

13 Wechsler D: Manual for the Wechsler Adult Intelligence Scale - Third Edition. San Antonio, The Psychological Corporation, 1997.

14 Kaplan EF, Goodgalss H, Weintraub S: The Boston Naming Test. Philadelphia, Lea \& Febiger, 1983.

15 Benton AL, Hamsher KS: Multilingual Aphasia Examination Manual. Iowa City, University of Iowa, 1976.

16 Wechsler D: Wechsler Memory Scale - Third Edition. San Antonio, The Psychological Corporation, 1997.

17 Delis DC, Kramer JH, Kaplan E, Ober BA: The California Verbal Learning Test. San Antonio, Psychological Corporation, 1987.

18 Delis DC, Kramer JH, Kaplan E, Ober BA: California Verbal Learning Test, ed 2. San Antonio, Psychological Corporation, 2000.

19 Osterrieth PA: Le test de copie d'une figure complexe. Arch Psychol 1944;30:206-356.

-20 Stroop JR: Studies in interference of serial verbal reactions. J Exp Psychol 1935;18:643-662.

21 Reitan RM, Wolfson D: The Halstead-Reitan Neuropsychological Test Battery: Therapy and Clinical Interpretation. Tucson, Neuropsychological Press, 1985.

22 Heaton RK: Wisconsin Card Sorting Test Manual. Odessa, Psychological Assessment Resources, Inc., 1981.

23 Collins DL, Neelin P, Peters TM, Evans AC: Automatic 3D intersubject registration of MR volumetric data in standardized Talairach space. J Comput Assist Tomogr 1994;18:192-205. 
24 Mazziotta J, Toga A, Evans A, Fox P, Lancaster J, Zilles K, Woods R, Paus T, Simpson G, Pike B, Holmes C, Collins L, Thompson P, MacDonald D, Iacoboni M, Schormann T, Amunts K, Palomero-Gallagher N, Geyer S, Parsons L, Narr K, Kabani N, Le Goualher G, Boomsma D, Cannon T, Kawashima R, Mazoyer B: A probabilistic atlas and reference system for the human brain: International Consortium for Brain Mapping (ICBM). Philos Trans $\mathrm{R}$ Soc Lond B Biol Sci 2001;356:1293-1322.

-25 Shattuck DW, Sandor-Leahy SR, Schaper KA, Rottenberg DA, Leahy RM: Magnetic resonance image tissue classification using a partial volume model. Neuroimage 2001;13:856-876.

26 Sowell ER TP, Mega MS, Zoumalan CI, Lindshield C, Rex DE: Gyral pattern delineation in 3D: surface curve protocol. 2000. www.loni.ucla.edu/ esowell/new_sulcvar.html.

27 Thompson PM, Toga AW: A surface-based technique for warping 3D images of the brain. IEEE Trans Med Imaging 1996;15:1-16.

28 Narr KL, Toga AW, Szeszko P, Thompson PM, Woods RP, Robinson D, Sevy S, Wang Y, Schrock K, Bilder RM: Cortical thinning in cingulate and occipital cortices in first episode schizophrenia. Biol Psychiatry 2005;58: $32-40$.

29 Thompson PM, Hayashi KM, de Zubicaray G, Janke AL, Rose SE, Semple J, Herman D, Hong MS, Dittmer SS, Doddrell DM, Toga AW: Dynamics of gray matter loss in Alzheimer's disease. J Neurosci 2003;23:994-1005.

-30 Luders E, Thompson PM, Narr KL, Toga AW, Jancke L, Gaser C: A curvature-based approach to estimate local gyrification on the cortical surface. Neuroimage 2006;29:1224-1230.

-31 Ewers M, Insel P, Jagust WJ, Shaw L, Trojanowski JJ, Aisen P, Petersen RC, Schuff N, Weiner MW: CSF biomarker and PIB-PET-derived beta-amyloid signature predicts metabolic, gray matter, and cognitive changes in nondemented subjects. Cereb Cortex 2012;22:1993-2004. 